\title{
Optimal realizations of floating-point implemented digital controllers with finite word length considerations
}

\author{
JUN WU†, SHENG CHEN‡*, JAMES F. WHIDBORNE§ and JIAN CHU† \\ The closed-loop stability issue of finite word length (FWL) realizations is investigated for digital controllers implemented \\ in floating-point arithmetic. Unlike the existing methods which only address the effect of the mantissa bits in floating- \\ point implementation to the sensitivity of closed-loop stability, the sensitivity of closed-loop stability is analysed with \\ respect to both the mantissa and exponent bits of floating-point implementation. A computationally tractable FWL \\ closed-loop stability measure is then defined, and the method of computing the value of this measure is given. The \\ optimal controller realization problem is posed as searching for a floating-point realization that maximizes the proposed \\ FWL closed-loop stability measure, and a numerical optimization technique is adopted to solve for the resulting \\ optimization problem. Simulation results show that the proposed design procedure yields computationally efficient \\ controller realizations with enhanced FWL closed-loop stability performance.
}

\section{Introduction}

The classical digital controller design methodology often assumes that the controller is implemented exactly, even though in reality a control law can only be realized in finite precision. It may seem that the uncertainty resulting from finite-precision implementation of the digital controller is so small, compared to the uncertainty within the plant, that this controller 'uncertainty' can simply be ignored. Increasingly, however, researchers have realized that this is not necessarily the case. Due to the finite word length (FWL) effect, a casual controller implementation may degrade the designed closedloop performance or even destabilize the designed stable closed-loop system, if the controller implementation structure is not carefully chosen. The effects of finiteprecision implementation have become more critical with the growing popularity of robust controller design methods which focus only on dealing with large plant uncertainty (Keel and Bhattacharryya 1997, Istepanian and Whidborne 2001). Generally speaking, there are two types of FWL errors in the digital controller. The first one is perturbation of controller parameters implemented with FWL and the second one is the rounding errors that occur in arithmetic operations of signals. Typically, effects of these two types of errors are investigated separately for the reason of mathematical tractability. The first type of FWL error directly concerns the

Received 1 August 2003. Revised 30 January 2004.

* Author for correspondence. e-mail: sqc@ecs.soton.ac.uk

$\dagger$ National Key Laboratory of Industrial Control Technology, Institute of Advanced Process Control, Zhejiang University, Hangzhou 310027, P. R. China.

$\downarrow$ School of Electronics and Computer Science, University of Southampton, Highfield, Southampton SO17 1BJ, UK.

$\S$ Department of Aerospace Sciences, School of Engineering, Cranfield University, Bedfordshire MK430AL, UK. critical issue of closed-loop stability, and many studies have investigated some closed-loop stability robustness measures, especially for fixed-point implementation (Fialho and Georgiou 1994, 2001, Madievski et al. 1995, Li 1998, Chen et al. 1999, Whidborne et al. 2000, 2001, Wu et al. $2001 \mathrm{a}, \mathrm{b})$. The second type of FWL error can also lead to instability through bounded limit cycles or floating-point unbounded responses and how to erase its effect on stability is the focus of the work of many researchers in control or digital filter system designs (Liu and Kaneko 1969, Kaneko 1973, Miller et al. 1988, 1989, Bauer and Wang 1993, Bauer 1995). Even when it does not arouse unstable behaviour, the second type of FWL error can still degrade the system performance and the effect of this is usually measured and studied with the so-called roundoff noise gain (Moroney et al. 1980, Williamson and Kadiman 1989, Li and Gevers 1990, Liu et al. 1992, Li et al. 2002).

Most works for FWL controller design adopt an indirect strategy, which relies on the following property. A control law can be implemented with different realizations, and these different realizations are all equivalent if they are implemented in infinite precision. However, different controller realizations possess different degrees of robustness to FWL errors. The control law is assumed to be given by some controller design methods, which may not take into account FWL considerations, and the FWL design is to select optimal realizations for the given control law by optimizing some FWL criteria. An alternative but better approach is to explicitly incorporate the FWL issues into the controller design process. For example, in the work of Liu et al. (1992), an FWL-LQG performance index was used to describe the LQG performance under FWL environment, and a fixed-order controller realization design method was presented to minimize this FWL-LQG 
cost function. This direct strategy should be a preferred approach, since it does not make specific assumptions on the controller. However, how to extend the idea of Liu et al. (1992) to various controller design methods is still an open problem. But this difficulty does not exist in the indirect strategy where controller synthesis and controller realization are two separate steps. Various existing controller design methods can be used to attain a transfer function or an initial realization of the controller, which can then be optimized to satisfy FWL implementation requirements.

In real-time applications where computational efficiency is critical, a digital controller implemented with fixed-point arithmetic has some advantages over floatingpoint format. However, the detrimental FWL effects are markedly increased in fixed-point implementation due to a reduced precision. It is therefore not surprising that previous works have focused on finding optimal controller realizations using fixed-point arithmetic by optimizing some FWL measures (Li and Gevers 1990, Liu et al. 1992, Gevers and $\mathrm{Li}$ 1993, Fialho and Georgiou 1994, 2001, Madievski et al. 1995, Li 1998, Chen et al. 1999, Whidborne et al. 2000, 2001, Wu et al. $2001 \mathrm{a}, \mathrm{b}, \mathrm{Li}$ et al. 2002). In all the previous works using fixed-point arithmetic, various measures, which can be shown to link to the bits required in implementing the fractional part of fixed-point representation, are optimized to produce optimal realizations. However, the dynamic range of fixed-point representation is determined by its integer part. Overflow occurs when there are not enough bits for the integer part. Optimizing these measures, while minimizing the bits required for the fractional part, may actually increase the bits required for the integer part. Arguably, a better approach would be to consider some measure which has a direct link to the total bit length required.

With a decrease in price and increase in availability, the use of floating-point processors in controller implementations has increased dramatically. Floatingpoint representation has quite different characteristics from fixed-point representation. The dynamic range of floating-point representation is determined by its exponent part. Overflow or underflow occurs when the bits for the exponent part are not sufficient. The effects of finite-precision floating-point implementation have been well studied in digital filter designs (Kalliojärvi and Astola 1996, Rao 1996, Ralev and Bauer 1999). However, there has been relatively little work studying explicitly floating-point digital controller implementations. Some exceptions include Rink and Chong (1979), Molchanov and Bauer (1995) and Whidborne and $\mathrm{Gu}$ (2002). In the work by Istepanian et al. (2000), a block-floating-point arithmetic was used, in which control coefficients were forced to have a common exponent and the problem was converted into a fixed-point one. The work by Whidborne and $\mathrm{Gu}$ (2002) represents a case of true floating-point implementation. In this work, a weighted closed-loop eigenvalue sensitivity index was defined for floating-point digital controller realizations. This index, however, only considers the mantissa part of floating-point arithmetic, under an assumption that the exponent bits are unlimited.

This paper adopts an indirect approach to consider the FWL parameter errors of floating-point implemented controllers. The generic contribution of this paper is to derive a new FWL closed-loop stability measure that explicitly considers both the mantissa and exponent parts of floating-point arithmetic. The remainder of this paper is organized as follows. Section 2 briefly summarizes the floating-point representation and highlights the multiplicative nature of perturbations resulting from FWL floating-point arithmetic. Section 3 analyses the FWL effect of floating-point arithmetic on closed-loop stability and addresses how to measure such an effect on floating-point implemented digital controllers. Section 4 defines a computationally tractable FWL closed-loop stability measure for floating-point controller realizations and provides the method of computing its value. In $\S 5$, the optimal floating-point controller realization problem is formulated, and a numerical optimization technique is adopted to solve the resulting optimization problem. Two examples are given in $\S 6$ to demonstrate the effectiveness of the proposed design method. Section 7 presents a brief discussion on the direct approach of Liu et al. (1992) and points out that the studies on optimizing FWL realizations for a fixed control law, such as this work, are helpful to explore the possible way of extending the idea of Liu et al. (1992). The paper concludes with $\S 8$.

\section{Floating-point representation}

Let the floor function $\lfloor x\rfloor$ denote the largest integer less than or equal to real number $x$. It is well known that any real number $x \in \mathcal{R}$ can be represented uniquely by

$$
x=(-1)^{s} \times w \times 2^{e}
$$

where $s \in\{0,1\}$ is for the sign of $x, w \in[0.5,1)$ is the mantissa of $x, e=\left\lfloor\log _{2}|x|\right\rfloor+1 \in \mathcal{Z}$ is the exponent of $x$ with $\mathcal{Z}$ denoting the set of integers. When $x$ is stored in a digital computer of finite $\beta$ bits in a floating-point format, the bits consist of three parts: one bit for $s, \beta_{w}$ bits for $w$ and $\beta_{e}$ bits for $e$. Obviously,

$$
\beta=1+\beta_{w}+\beta_{e} .
$$

As the finite $\beta_{e}$ bits can only support a limited exponent range, we define $\underline{e}$ and $\bar{e}$ to represent the lower and upper limits of the exponent range, respectively, and denote the exponent range that is supported by $\beta_{e}$ bits as

$$
\mathcal{Z}_{[\underline{e}, \bar{e}]} \triangleq\{e \mid e \in \mathcal{Z}, \underline{e} \leq e \leq \bar{e}\} .
$$


In fact, the exponent range $\mathcal{Z}_{[e, \bar{e}]}$ depends not only on $\beta_{e}$ but also on the set of real numbers which is to be represented. As an example, consider the set of three numbers $\left\{0.7 \times 2^{-1},-0.9 \times 2,0.8 \times 2^{2}\right\}$. At least two bits are required to describe their exponents, with 00 representing $-1,01$ for 0,10 representing 1 and 11 for 2. Thus, $\underline{e}=-1, \bar{e}=2$ and $\mathcal{Z}_{[-1,2]}=\{-1,0,1,2\}$ are determined by the three numbers represented in this example of exponent bits $\beta_{e}=2$. Obviously

$$
\bar{e}-\underline{e}=2^{\beta_{e}}-1 .
$$

Overflow and underflow can occur in floating-point arithmetic of FWL. Overflow occurs when a floating-point scheme with $\mathcal{Z}_{[\underline{e}, \bar{e}]}$ is used to represent a real number whose exponent is greater than $\bar{e}$, while underflow occurs when a floating-point scheme with $\mathcal{Z}_{[\underline{e}, \bar{e}]}$ is used to represent a real number whose exponent is smaller than $\underline{e}$. It should be emphasized that in many practical problems, the problem objective function is highly sensitive to small parameter perturbation and, therefore, small numbers should not simply be 'underflowed' to zero. For a demonstration, we refer to the so-called fragility issue (Keel and Bhattacharryya 1997). In floatingpoint arithmetic with FWL, underflow should generally be treated as seriously as overflow, and avoided if possible.

Since $\beta_{w}$ and $\beta_{e}$ are finite, the set of numbers that is represented by a particular floating-point scheme is not dense on the real line. Thus the set of possible floatingpoint numbers is given by

$$
\begin{aligned}
\mathcal{F} \triangleq & \left\{(-1)^{s}\left(0.5+\sum_{i=1}^{\beta_{w}} b_{i} 2^{-(i+1)}\right) 2^{e}:\right. \\
& \left.s \in\{0,1\}, b_{i} \in\{0,1\}, e \in \mathcal{Z}_{[\underline{e}, \bar{e}]}\right\} \cup\{0\} .
\end{aligned}
$$

When no underflow or overflow occurs, that is, the exponent of $x$ is within $\mathcal{Z}_{[\underline{\underline{\mathcal{e}}}, \bar{e}]}$, the floating-point quantization operator $\mathcal{Q}: \mathcal{R} \rightarrow \overrightarrow{\mathcal{F}}$ can be defined as

$\mathcal{Q}(x) \triangleq \begin{cases}\operatorname{sgn}(x) 2^{\left(e-\beta_{w}-1\right)}\left\lfloor 2^{\left(\beta_{w}-e+1\right)}|x|+0.5\right\rfloor, & \text { for } x \neq 0 \\ 0, & \text { for } x=0 .\end{cases}$

In the above definition, magnitude rounding is used as the mantissa quantization format. Define the quantization error $\varepsilon$ as

$$
\varepsilon \triangleq|x-\mathcal{Q}(x)|
$$

Then

$$
\begin{aligned}
\varepsilon & =|\operatorname{sgn}(x)| x\left|-\operatorname{sgn}(x) 2^{\left(e-\beta_{w}-1\right)}\left\lfloor 2^{\left(\beta_{w}-e+1\right)}|x|+0.5\right\rfloor\right| \\
& =2^{\left(e-\beta_{w}-1\right)}\left|2^{\left(\beta_{w}-e+1\right)}\right| x\left|-\left\lfloor 2^{\left(\beta_{w}-e+1\right)}|x|+0.5\right\rfloor\right| \\
& \leq 2^{\left(e-\beta_{w}-1\right)} \times 0.5 .
\end{aligned}
$$

From the definition of the exponent $e$, we have

$$
2^{e} \times 0.5=2^{\left\lfloor\log _{2}|x|\right\rfloor} \leq 2^{\log _{2}|x|}=|x| .
$$

Combining (8) and (9) leads to

$$
\varepsilon \leq|x| 2^{-\left(\beta_{w}+1\right)} .
$$

Thus, when $x$ is implemented in floating-point format of $\beta_{w}$ mantissa bits, assuming no underflow or overflow, it can be seen from (7) and (10) that $x$ is perturbed to

$$
\mathcal{Q}(x)=x(1+\delta), \quad|\delta| \leq 2^{-\left(\beta_{w}+1\right)} .
$$

Clearly, the perturbation resulting from finite-precision floating-point arithmetic is multiplicative, unlike the perturbation resulting from finite-precision fixed-point arithmetic, which is additive.

\section{Problem statement}

Consider the discrete-time closed-loop control system, consisting of a linear time invariant plant $P(z)$ and a digital controller $C(z)$. The plant model $P(z)$ is assumed to be strictly proper with a state-space description $\left(\mathbf{A}_{P}, \mathbf{B}_{P}, \mathbf{C}_{P}\right)$, where $\mathbf{A}_{P} \in \mathcal{R}^{m \times m}, \mathbf{B}_{P} \in \mathcal{R}^{m \times l}$ and $\mathbf{C}_{P} \in \mathcal{R}^{q \times m}$. Let $\left(\mathbf{A}_{C}, \mathbf{B}_{C}, \mathbf{C}_{C}, \mathbf{D}_{C}\right)$ be a state-space description of the controller $C(z)$, with $\mathbf{A}_{C} \in \mathcal{R}^{n \times n}$, $\mathbf{B}_{C} \in \mathcal{R}^{n \times q}, \mathbf{C}_{C} \in \mathcal{R}^{l \times n}$ and $\mathbf{D}_{C} \in \mathcal{R}^{l \times q}$. A linear system with a given transfer function matrix has an infinite number of state-space descriptions. In fact, if $\left(\mathbf{A}_{C}^{0}\right.$, $\left.\mathbf{B}_{C}^{0}, \mathbf{C}_{C}^{0}, \mathbf{D}_{C}^{0}\right)$ is a state-space description of $C(z)$, all the state-space descriptions of $C(z)$ form a realization set

$$
\begin{aligned}
\mathcal{S}_{C} \triangleq\left\{\left(\mathbf{A}_{C}, \mathbf{B}_{C}, \mathbf{C}_{C}, \mathbf{D}_{C}\right) \mid \mathbf{A}_{C}=\mathbf{T}^{-1} \mathbf{A}_{C}^{0} \mathbf{T},\right. \\
\left.\mathbf{B}_{C}=\mathbf{T}^{-1} \mathbf{B}_{C}^{0}, \mathbf{C}_{C}=\mathbf{C}_{C}^{0} \mathbf{T}, \mathbf{D}_{C}=\mathbf{D}_{C}^{0}\right\}
\end{aligned}
$$

where the transformation matrix $\mathbf{T} \in \mathcal{R}^{n \times n}$ is an arbitrary non-singular matrix. Denote

$$
\mathbf{X}=\left[x_{j, k}\right] \triangleq\left[\begin{array}{cc}
\mathbf{D}_{C} & \mathbf{C}_{C} \\
\mathbf{B}_{C} & \mathbf{A}_{C}
\end{array}\right] .
$$

The stability of the closed-loop control system depends on the eigenvalues of the closed-loop transition matrix

$$
\begin{aligned}
\overline{\mathbf{A}}(\mathbf{X}) & =\left[\begin{array}{cc}
\mathbf{A}_{P}+\mathbf{B}_{P} \mathbf{D}_{C} \mathbf{C}_{P} & \mathbf{B}_{P} \mathbf{C}_{C} \\
\mathbf{B}_{C} \mathbf{C}_{P} & \mathbf{A}_{C}
\end{array}\right] \\
& =\left[\begin{array}{cc}
\mathbf{A}_{P} & \mathbf{0} \\
\mathbf{0} & \mathbf{0}
\end{array}\right]+\left[\begin{array}{cc}
\mathbf{B}_{P} & \mathbf{0} \\
\mathbf{0} & \mathbf{I}_{n}
\end{array}\right] \mathbf{X}\left[\begin{array}{cc}
\mathbf{C}_{P} & \mathbf{0} \\
\mathbf{0} & \mathbf{I}_{n}
\end{array}\right] \\
& \triangleq \mathbf{M}_{0}+\mathbf{M}_{1} \mathbf{X} \mathbf{M}_{2}
\end{aligned}
$$

where $\mathbf{0}$ denotes the zero matrix of appropriate dimension and $\mathbf{I}_{n}$ the $n \times n$ identity matrix. All the different realizations $\mathbf{X}$ in $\mathcal{S}_{C}$ have exactly the same set of closedloop poles if they are implemented with infinite precision. Since the closed-loop system has been designed to be stable, all the eigenvalues $\lambda_{i}(\overline{\mathbf{A}}(\mathbf{X})), 1 \leq i \leq m+n$, are within the unit disk. Define

$$
\|\mathbf{X}\|_{\max } \triangleq \max _{j, k}\left|x_{j, k}\right|
$$


and

$$
g(\mathbf{X}) \triangleq \min _{j, k}\left\{\left|x_{j, k}\right|: x_{j, k} \neq 0\right\} .
$$

The controller $\mathbf{X}$ is implemented with a floating-point processor of $\beta_{e}$ exponent bits, $\beta_{w}$ mantissa bits and one sign bit.

First, in order to avoid underflow and/or overflow, both the exponent of $\|\mathbf{X}\|_{\max }$ and the exponent of $g(\mathbf{X})$ should be within $\mathcal{Z}_{[e, \bar{e}]}$ supported by the $\beta_{e}$ exponent bits. We define an exponent measure for the floatingpoint controller realization $\mathbf{X}$ as

$$
\gamma(\mathbf{X}) \triangleq \log _{2}\left(\frac{4\|\mathbf{X}\|_{\max }}{g(\mathbf{X})}\right) .
$$

The rationale of this exponent measure becomes clear in the following (obvious) proposition.

Proposition 1: $\mathbf{X}$ can be represented in the floatingpoint format of $\beta_{e}$ exponent bits without underflow or overflow, if

$$
2^{\beta_{e}} \geq \log _{2}\left(\frac{\|\mathbf{X}\|_{\max }}{g(\mathbf{X})}\right)+2 .
$$

Let $\beta_{e}^{\min }$ be the smallest exponent bit length that, when used to implement $\mathbf{X}$, can avoid underflow and overflow. It can be computed as

$$
\beta_{e}^{\min }=-\left\lfloor-\log _{2}\left(\left\lfloor\log _{2}\|\mathbf{X}\|_{\max }\right\rfloor-\left\lfloor\log _{2} g(\mathbf{X})\right\rfloor+1\right)\right\rfloor .
$$

The measure $\gamma(\mathbf{X})$ provides an estimate of $\beta_{e}^{\min }$ as

$$
\hat{\beta}_{e}^{\min } \triangleq-\left\lfloor-\log _{2} \gamma(\mathbf{X})\right\rfloor .
$$

It is clear that $\hat{\beta}_{e}^{\min } \geq \beta_{e}^{\min }$.

Second, when there is no underflow or overflow, according to (11), $\mathbf{X}$ is perturbed to $\mathbf{X}+\mathbf{X} \circ \boldsymbol{\Delta}$ due to the effect of finite $\beta_{w}$ where

$$
\mathbf{X} \circ \Delta \triangleq\left[x_{j, k} \delta_{j, k}\right]
$$

represents the Hadamard product of $\mathbf{X}$ and $\boldsymbol{\Delta}=\left[\delta_{j, k}\right]$. Each element of $\Delta$ is bounded by $\pm 2^{-\left(\beta_{w}+1\right)}$, that is

$$
\|\boldsymbol{\Delta}\|_{\max } \leq 2^{-\left(\beta_{w}+1\right)} .
$$

With the perturbation $\Delta, \lambda_{i}(\overline{\mathbf{A}}(\mathbf{X}))$ is moved to $\lambda_{i}(\overline{\mathbf{A}}(\mathbf{X}+\mathbf{X} \circ \Delta))$. If an eigenvalue of $\overline{\mathbf{A}}(\mathbf{X}+\mathbf{X} \circ \boldsymbol{\Delta})$ is outside the open unit disk, the closed-loop system, designed to be stable, becomes unstable with the finiteprecision floating-point implemented $\mathbf{X}$.

It is therefore critical to know when the FWL error will cause closed-loop instability. This means that we would like to know the largest open 'hypercube' in the perturbation space, within which the closed-loop system remains stable. Based on this consideration, a mantissa measure for the floating-point realization $\mathbf{X}$ can be defined as

$$
\mu_{0}(\mathbf{X}) \triangleq \inf \left\{\|\boldsymbol{\Delta}\|_{\max }: \overline{\mathbf{A}}(\mathbf{X}+\mathbf{X} \circ \boldsymbol{\Delta}) \text { is unstable }\right\} .
$$

From the above definition, the following proposition is obvious.

Proposition 2: $\overline{\mathbf{A}}(\mathbf{X}+\mathbf{X} \circ \boldsymbol{\Delta})$ is stable if $\|\boldsymbol{\Delta}\|_{\max }<$ $\mu_{0}(\mathbf{X})$.

Let $\beta_{w}^{\min }$ be the mantissa bit length such that $\forall \beta_{w} \geq \beta_{w}^{\min }, \overline{\mathbf{A}}(\mathbf{X}+\mathbf{X} \circ \Delta)$ is stable for the floatingpoint implemented $\mathbf{X}$ with $\beta_{w}$ mantissa bits and $\overline{\mathbf{A}}(\mathbf{X}+\mathbf{X} \circ \boldsymbol{\Delta})$ is unstable for the floating-point implemented $\mathbf{X}$ with $\beta_{w}^{\min }-1$ mantissa bits. Except through simulation, $\beta_{w}^{\min }$ is generally unknown. It should be pointed out that due to the complex non-linear relationship between $\beta_{w}$ and closed-loop stability, there may exist some odd cases of smaller mantissa bit length $\beta_{w}<\beta_{w}^{\min }-1$ which regain closed-loop stability. For example, consider the stable closed-loop system containing the plant

$$
P(z)=\frac{-1.66(z-1.2)(z-1.1)(z-0.4)}{\left(z^{2}-0.35 z+0.49\right)(z+4)}
$$

and the controller $C(z)=K=0.66$. When $\beta_{w} \geq 4$, the closed-loop system with the FWL implemented $K$ is stable, but it becomes unstable with $\beta_{w}=3$ where the implemented value of $K$ is 0.6875 . However, the closedloop regains stability with $\beta_{w}=2$ where the implemented value of $K$ is 0.625 . The system becomes unstable again for $\beta_{w}=1$ where the implemented value of $K$ is 0.75 . Figure 1 shows the root locus plot of this threeorder system which gives the closed-loop pole positions for all values of $K$. From figure 1, it can be seen that the system is unstable when the implemented value of $K$ is greater than 0.686 or less than 0.513 . For this system, $\beta_{w}^{\min }$ is 4 rather than 2 . The mantissa measure $\mu_{0}(\mathbf{X})$ provides an estimate of $\beta_{w}^{\min }$ as

$$
\hat{\boldsymbol{\beta}}_{w 0}^{\min } \triangleq-\left\lfloor\log _{2} \mu_{0}(\mathbf{X})\right\rfloor-1 .
$$

It can be seen that $\hat{\beta}_{w 0}^{\min } \geq \beta_{w}^{\min }$.

Define the minimum total bit length required in floating-point implementation as

$$
\beta^{\min } \triangleq \beta_{e}^{\min }+\beta_{w}^{\min }+1 .
$$

Clearly, a floating-point implemented $\mathbf{X}$ with a bit length $\beta \geq \beta^{\min }$ can guarantee no underflow, no overflow and closed-loop stability. Combining the measures $\gamma(\mathbf{X})$ and $\mu_{0}(\mathbf{X})$ results in the following true FWL closed-loop stability measure for the floating-point realization $\mathbf{X}$

$$
\rho_{0}(\mathbf{X}) \triangleq \mu_{0}(\mathbf{X}) / \gamma(\mathbf{X}) .
$$

An estimate of $\beta^{\min }$ is given by $\rho_{0}(\mathbf{X})$ as

$$
\hat{\beta}_{0}^{\min } \triangleq-\left\lfloor\log _{2} \rho_{0}(\mathbf{X})\right\rfloor+1 .
$$




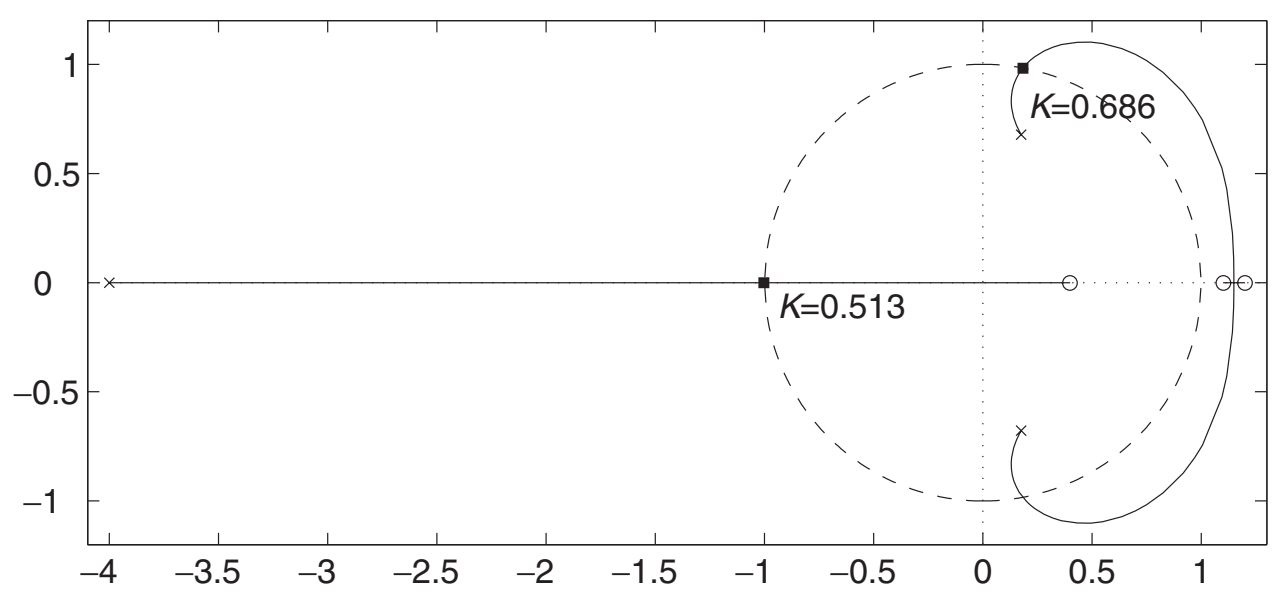

Figure 1. Root locus plot of a three-order system.

It is clear that $\hat{\beta}_{0}^{\min } \geq \beta^{\mathrm{min}}$. The following proposition summarizes the usefulness of $\rho_{0}(\mathbf{X})$ as a measure for the FWL characteristics of $\mathbf{X}$.

Proposition 3: A floating-point implemented $\mathbf{X}$ with a bit length $\beta$ can guarantee no underflow, no overflow and closed-loop stability, if

$$
2^{\beta-1} \geq \frac{1}{\rho_{0}(\mathbf{X})} .
$$

Since the closed-loop stability measure $\rho_{0}(\mathbf{X})$ is a function of the controller realization $\mathbf{X}$ and $\hat{\boldsymbol{\beta}}_{0}^{\min }$ decreases with the increase of $\rho_{0}(\mathbf{X})$, an optimal realization can in theory be found by maximizing $\rho_{0}(\mathbf{X})$, leading to the optimal controller realization problem

$$
v_{\text {true }} \triangleq \max _{\mathbf{X} \in \mathcal{S}_{C}} \rho_{0}(\mathbf{X})
$$

However, the difficulty with this approach is that computing the value of $\mu_{0}(\mathbf{X})$ is an unsolved open problem. Thus, the true FWL closed-loop stability measure $\rho_{0}(\mathbf{X})$ and the optimal realization problem (28) have limited practical significance. In the next section, we will seek an alternative measure that can not only quantify FWL characteristics of $\mathbf{X}$ but is also computationally tractable.

\section{A tractable FWL closed-loop stability measure}

When the FWL error $\Delta$ is small, from a first-order approximation, $\forall i \in\{1, \ldots, m+n\}$

$$
\left|\lambda_{i}(\overline{\mathbf{A}}(\mathbf{X}+\mathbf{X} \circ \Delta))\right|-\left.\left|\lambda_{i}(\overline{\mathbf{A}}(\mathbf{X}))\right| \approx \sum_{j=1}^{l+n} \sum_{k=1}^{q+n} \frac{\partial\left|\lambda_{i}\right|}{\partial \delta_{j, k}}\right|_{\Delta=0} \delta_{j, k} .
$$

For the derivative matrix $\partial\left|\lambda_{i}\right| / \partial \boldsymbol{\Delta}=\left[\partial\left|\lambda_{i}\right| / \partial \delta_{j, k}\right]$, define

$$
\left\|\frac{\partial\left|\lambda_{i}\right|}{\partial \boldsymbol{\Delta}}\right\|_{\text {sum }} \triangleq \sum_{j, k}\left|\frac{\partial\left|\lambda_{i}\right|}{\partial \delta_{j, k}}\right| .
$$

Then

$$
\left|\lambda_{i}(\overline{\mathbf{A}}(\mathbf{X}+\mathbf{X} \circ \boldsymbol{\Delta}))\right|-\left|\lambda_{i}(\overline{\mathbf{A}}(\mathbf{X}))\right| \leq\|\boldsymbol{\Delta}\|_{\max }\left\|\left.\frac{\partial\left|\lambda_{i}\right|}{\partial \boldsymbol{\Delta}}\right|_{\boldsymbol{\Delta}=0}\right\|_{\text {sum }} .
$$

This leads to the following mantissa measure for the floating-point realization $\mathbf{X}$

$$
\mu_{1}(\mathbf{X}) \triangleq \min _{i \in\{1, \ldots, m+n\}} \frac{1-\left|\lambda_{i}(\overline{\mathbf{A}}(\mathbf{X}))\right|}{\left\|\partial\left|\lambda_{i}\right| /\left.\partial \Delta\right|_{\Delta=0}\right\|_{\text {sum }}} .
$$

For those FWL errors that make (31) hold, if $\|\boldsymbol{\Delta}\|_{\max }<\mu_{1}(\mathbf{X})$, then $\left|\lambda_{i}(\overline{\mathbf{A}}(\mathbf{X}+\mathbf{X} \circ \boldsymbol{\Delta}))\right|<1$ which means that the closed-loop remains stable under the FWL error $\Delta$. In other words, the closed-loop can tolerate those FWL perturbations $\boldsymbol{\Delta}$ whose norms $\|\boldsymbol{\Delta}\|_{\max }$ are less than $\mu_{1}(\mathbf{X})$. The larger $\mu_{1}(\mathbf{X})$ is, the larger FWL errors the closed-loop system can tolerate. Similar to (23), from the mantissa measure $\mu_{1}(\mathbf{X})$, an estimate of $\beta_{w}^{\min }$ is given as

$$
\hat{\beta}_{w 1}^{\min } \triangleq-\left\lfloor\log _{2} \mu_{1}(\mathbf{X})\right\rfloor-1 .
$$

The assumption of small $\Delta$ is usually valid in floatingpoint implementation. Generally speaking, there is no rigorous relationship between $\mu_{0}(\mathbf{X})$ and $\mu_{1}(\mathbf{X})$, but $\mu_{1}(\mathbf{X})$ is connected with a lower bound of $\mu_{0}(\mathbf{X})$ in some ways: there are 'stable perturbation hypercubes' larger than $\left\{\boldsymbol{\Delta}:\|\boldsymbol{\Delta}\|_{\max }<\mu_{1}(\mathbf{X})\right\}$ while there is no 'stable perturbation hypercube' larger than $\left\{\boldsymbol{\Delta}:\|\boldsymbol{\Delta}\|_{\max }<\mu_{0}(\mathbf{X})\right\}$ (Wu et al. 2000, 2001 a). Hence, in most cases, it is reasonable to take that $\mu_{1}(\mathbf{X}) \leq \mu_{0}(\mathbf{X})$ and $\hat{\boldsymbol{\beta}}_{w 1}^{\min } \geq \hat{\boldsymbol{\beta}}_{w 0}^{\min }$. More importantly, unlike the measure 
$\mu_{0}(\mathbf{X})$, the value of $\mu_{1}(\mathbf{X})$ can be computed explicitly. It is easy to see that

$$
\left.\frac{\partial\left|\lambda_{i}\right|}{\partial \boldsymbol{\Delta}}\right|_{\Delta=0}=\frac{\partial\left|\lambda_{i}\right|}{\partial \mathbf{X}} \circ \mathbf{X}
$$

Let $\mathbf{p}_{i}$ be a right eigenvector of $\overline{\mathbf{A}}(\mathbf{X})$ corresponding to the eigenvalue $\lambda_{i}$. Define

$$
\mathbf{M}_{\mathbf{p}} \triangleq\left[\begin{array}{llll}
\mathbf{p}_{1} & \mathbf{p}_{2} & \cdots & \mathbf{p}_{m+n}
\end{array}\right]
$$

and

$$
\mathbf{M}_{\mathbf{y}} \triangleq\left[\begin{array}{llll}
\mathbf{y}_{1} & \mathbf{y}_{2} & \cdots & \mathbf{y}_{m+n}
\end{array}\right]=\mathbf{M}_{\mathbf{p}}^{-\mathrm{H}}
$$

where the superscript $\mathrm{H}$ denotes the conjugate transpose operator and $\mathbf{y}_{i}$ is called the reciprocal left eigenvector related to $\mathbf{p}_{i}$. The following lemma is due to $\mathrm{Li}$ (1998).

Lemma 1: Let $\overline{\mathbf{A}}(\mathbf{X})=\mathbf{M}_{0}+\mathbf{M}_{1} \mathbf{X} \mathbf{M}_{2}$ given in (14) be diagonalizable. Then

$$
\frac{\partial \lambda_{i}}{\partial \mathbf{X}}=\mathbf{M}_{1}^{\mathrm{T}} \mathbf{y}_{i}^{*} \mathbf{p}_{i}^{\mathrm{T}} \mathbf{M}_{2}^{\mathrm{T}}
$$

where the superscript $*$ denotes the conjugate operation and $\mathrm{T}$ the transpose operator.

Comments: The necessary and sufficient condition for $\overline{\mathbf{A}}(\mathbf{X})$ being diagonalizable is that it has $m+n$ linearly independent eigenvectors. This includes two cases. Firstly, $\overline{\mathbf{A}}(\mathbf{X})$ has $m+n$ distinct eigenvalues. In this case, we can differentiate eigenvalues simply by their values. Secondly, the eigenvalues of $\overline{\mathbf{A}}(\mathbf{X})$ are not all distinct but there are $m+n$ linearly independent eigenvectors. In this case, we can differentiate eigenvalues by their corresponding eigenvectors.

The following proposition shows that, given an $\mathbf{X}$, the value of $\mu_{1}(\mathbf{X})$ can easily be calculated.

Proposition 4: Let $\overline{\mathbf{A}}(\mathbf{X})$ be diagonalizable. Then

$$
\mu_{1}(\mathbf{X})=\min _{i \in\{1, \ldots, m+n\}} \frac{\left|\lambda_{i}\right|\left(1-\left|\lambda_{i}\right|\right)}{\left\|\left(\mathbf{M}_{1}^{\mathrm{T}} \operatorname{Re}\left[\lambda_{i}^{*} \mathbf{y}_{i}^{*} \mathbf{p}_{i}^{\mathrm{T}}\right] \mathbf{M}_{2}^{\mathrm{T}}\right) \circ \mathbf{X}\right\|_{\mathrm{sum}}} .
$$

Proof: Noting $\left|\lambda_{i}\right|=\sqrt{\lambda_{i}^{*} \lambda_{i}}$ leads to

$$
\begin{aligned}
\frac{\partial\left|\lambda_{i}\right|}{\partial \mathbf{X}} & =\frac{1}{2 \sqrt{\lambda_{i}^{*} \lambda_{i}}}\left(\frac{\partial \lambda_{i}^{*}}{\partial \mathbf{X}} \lambda_{i}+\lambda_{i}^{*} \frac{\partial \lambda_{i}}{\partial \mathbf{X}}\right) \\
& =\frac{1}{2\left|\lambda_{i}\right|}\left(\left(\frac{\partial \lambda_{i}}{\partial \mathbf{X}}\right)^{*} \lambda_{i}+\lambda_{i}^{*} \frac{\partial \lambda_{i}}{\partial \mathbf{X}}\right)=\frac{1}{\left|\lambda_{i}\right|} \operatorname{Re}\left[\lambda_{i}^{*} \frac{\partial \lambda_{i}}{\partial \mathbf{X}}\right] .
\end{aligned}
$$

Combining (32), (34), (39) and Lemma 1 results in this proposition.

Replacing $\mu_{0}(\mathbf{X})$ with $\mu_{1}(\mathbf{X})$ in (25) leads to a computationally tractable FWL closed-loop stability measure

$$
\rho_{1}(\mathbf{X}) \triangleq \mu_{1}(\mathbf{X}) / \gamma(\mathbf{X})
$$

From the above measure, an estimate of $\beta^{\min }$ is given as

$$
\hat{\beta}_{1}^{\min } \triangleq-\left\lfloor\log _{2} \rho_{1}(\mathbf{X})\right\rfloor+1 .
$$

Note that the computationally tractable mantissa measure (32) is related to the eigenvalue module sensitivities with respect to (w.r.t.) the controller perturbation. This is similar to the case of controller realizations implemented in fixed-point arithmetic, where an existing FWL precision measure is defined as (Wu et al. $2001 \mathrm{a}$ )

$$
\mu_{f}(\mathbf{X}) \triangleq \min _{i \in\{1, \ldots, m+n\}} \frac{1-\left|\lambda_{i}(\overline{\mathbf{A}}(\mathbf{X}))\right|}{\left\|\partial\left|\lambda_{i}\right| / \partial \mathbf{X}\right\|_{\text {sum }}} .
$$

The idea underpinning $\mu_{1}(\mathbf{X})$ in (32), namely the sensitivity w.r.t. controller perturbation, is the same as the sensitivity w.r.t. controller parameters that underpins $\mu_{f}(\mathbf{X})$ in (42). In fact, it is well known that with an FWL fixed-point implementation, $\mathbf{X}$ is perturbed to $\mathbf{X}+\boldsymbol{\Delta}$ and

$$
\left|\lambda_{i}(\overline{\mathbf{A}}(\mathbf{X}+\Delta))\right|-\left.\left|\lambda_{i}(\overline{\mathbf{A}}(\mathbf{X}))\right| \approx \sum_{j=1}^{l+n} \sum_{k=1}^{q+n} \frac{\partial\left|\lambda_{j}\right|}{\partial \delta_{j, k}}\right|_{\Delta=0} \delta_{j, k} .
$$

Obviously, in the fixed-point case, we have

$$
\left.\frac{\partial\left|\lambda_{i}\right|}{\partial \boldsymbol{\Delta}}\right|_{\boldsymbol{\Delta}=0}=\frac{\partial\left|\lambda_{i}\right|}{\partial \mathbf{X}}
$$

and the fixed-point FWL measure $\mu_{f}(\mathbf{X})$ can be written as

$$
\mu_{f}(\mathbf{X})=\min _{i \in\{1, \ldots, m+n\}} \frac{1-\left|\lambda_{i}(\overline{\mathbf{A}}(\mathbf{X}))\right|}{\left\|\partial\left|\lambda_{i}\right| /\left.\partial \boldsymbol{\Delta}\right|_{\Delta=0}\right\|_{\text {sum }}} .
$$

On the other hand, from (32) and (34), it can be seen that

$$
\mu_{1}(\mathbf{X})=\min _{i \in\{1, \ldots, m+n\}} \frac{1-\left|\lambda_{i}(\overline{\mathbf{A}}(\mathbf{X}))\right|}{\left\|\left(\partial\left|\lambda_{i}\right| / \partial \mathbf{X}\right) \circ \mathbf{X}\right\|_{\text {sum }}}
$$

which is clearly linked to the eigenvalue module sensitivities w.r.t. the controller parameters. The Hadamard product in (46) merely reflects the multiplicative characteristic of floating-point perturbations.

It is also useful to compare the proposed measure with the previous results for floating-point format, especially the most recent one given by Whidborne and $\mathrm{Gu}$ (2002). For a complex-valued matrix $\mathbf{Y}=\left[y_{j, k}\right]$, define the Frobenius norm

$$
\|\mathbf{Y}\|_{\mathrm{F}} \triangleq\left(\sum_{j, k} y_{j, k}^{*} y_{j, k}\right)^{1 / 2} .
$$

Under an assumption that the exponent bits are unlimited, the computationally tractable weighted closed-loop eigenvalue sensitivity index addressed in Whidborne and $\mathrm{Gu}$ (2002) is given by

$$
\Upsilon(\mathbf{X}) \triangleq \sum_{i=1}^{m+n} \alpha_{i} \Upsilon_{i}(\mathbf{X})
$$


where $\alpha_{i}$ are non-negative weighting scalars and $Y_{i}(\mathbf{X})$ are single-eigenvalue sensitivities defined by

$$
\Upsilon_{i}(\mathbf{X}) \triangleq\|\mathbf{X}\|_{\mathrm{F}}^{2}\left\|\frac{\partial \lambda_{i}}{\partial \mathbf{X}}\right\|_{\mathrm{F}}^{2} .
$$

The thinking behind the above definition is as follows. From a first-order approximation, it can easily be shown that

$$
\left|\lambda_{i}(\overline{\mathbf{A}}(\mathbf{X}+\mathbf{X} \circ \boldsymbol{\Delta}))-\lambda_{i}(\overline{\mathbf{A}}(\mathbf{X}))\right| \leq\|\boldsymbol{\Delta}\|_{\max }\|\mathbf{X}\|_{\mathrm{F}}\left\|\frac{\partial \lambda_{i}}{\partial \mathbf{X}}\right\|_{\mathrm{F}} .
$$

Therefore, for those multiplicative perturbations bounded by $\|\boldsymbol{\Delta}\|_{\max }$, a small $\Upsilon_{i}(\mathbf{X})$ will limit the resulting change of the corresponding eigenvalue within a small range.

The first obvious observation is that $\rho_{1}(\mathbf{X})$ considers both the mantissa and exponent of floating-point arithmetic and is therefore able to handle all the aspects of underflow, overflow and closed-loop stability, while $r(\mathbf{X})$ only considers the mantissa part of floating-point arithmetic and is thus 'incomplete'. Secondly, it can be seen that $r(\mathbf{X})$ deals with the sensitivity of $\lambda_{i}$ while $\rho_{1}(\mathbf{X})\left(\mu_{1}(\mathbf{X})\right)$ considers the sensitivity of $\left|\lambda_{i}\right|$. It is well known that the stability of a discrete-time linear timeinvariant system depends only on the moduli of its eigenvalues. As $Y(\mathbf{X})$ includes the unnecessary eigenvalue arguments in consideration, it is generally conservative in comparison with $\rho_{1}(\mathbf{X})$. Third, $\rho_{1}(\mathbf{X})$ uses $\left\|\left(\partial\left|\lambda_{i}\right| / \partial \mathbf{X}\right) \circ \mathbf{X}\right\|_{\text {sum }}$ while $r(\mathbf{X})$ uses $\|\mathbf{X}\|_{\mathrm{F}}\left\|\partial \lambda_{i} / \partial \mathbf{X}\right\|_{\mathrm{F}}$ in checking the change of an eigenvalue. It is easy to see that

$$
\begin{aligned}
\left|\lambda_{i}(\overline{\mathbf{A}}(\mathbf{X}+\mathbf{X} \circ \boldsymbol{\Delta}))\right|-\left|\lambda_{i}(\overline{\mathbf{A}}(\mathbf{X}))\right| & \leq\|\boldsymbol{\Delta}\|_{\max }\left\|\frac{\partial\left|\lambda_{i}\right|}{\partial \mathbf{X}} \circ \mathbf{X}\right\|_{\text {sum }} \\
& \leq\|\boldsymbol{\Delta}\|_{\max }\|\mathbf{X}\|_{\mathrm{F}}\left\|\frac{\partial \lambda_{i}}{\partial \mathbf{X}}\right\|_{\mathrm{F}} .
\end{aligned}
$$

Obviously, $\left\|\left(\partial\left|\lambda_{i}\right| / \partial \mathbf{X}\right) \circ \mathbf{X}\right\|_{\text {sum }}$ gives a more accurate limit than $\|\mathbf{X}\|_{\mathrm{F}}\left\|\partial \lambda_{i} / \partial \mathbf{X}\right\|_{\mathrm{F}}$ does on the change of the corresponding eigenvalue module due to the multiplicative perturbations. This again implies that $\rho_{1}(\mathbf{X})$ is less conservative than $Y(\mathbf{X})$ in estimating the robustness of closed-loop stability with respect to controller perturbations. The fourth observation is that $\rho_{1}(\mathbf{X})$ provides an estimate of $\beta^{\min }, \hat{\boldsymbol{\beta}}_{1}^{\min }$ in (41), while $\boldsymbol{Y}(\mathbf{X})$ cannot provide information on bit length to the designer. One reason is that the measure $\rho_{1}(\mathbf{X})$ consists of two components, with $\mu_{1}(\mathbf{X})$ addressing the stability margin and eigenvalue sensitivity linked to the mantissa bits and $\gamma(\mathbf{X})$ considering the exponent bits, while $r(\mathbf{X})$ only focuses on the eigenvalue sensitivity partially linked to the mantissa part. The other reason is that, over all the closed-loop eigenvalues, $\mu_{1}(\mathbf{X})$ considers the 'worst' one while $r(\mathbf{X})$ considers a 'weighted average'.
Finally, it is worth emphasizing that the generic idea of considering both the exponent, which defines the dynamic range, and mantissa, which defines the accuracy or precision, of the floating-point arithmetic is a sensible one and should be extended to other situations where different representation schemes, such as fixedpoint format, are used.

\section{Optimization procedure}

As different realizations $\mathbf{X}$ have different values of the FWL closed-loop stability measure $\rho_{1}(\mathbf{X})$, it is of practical importance to find an 'optimal' realization $\mathbf{X}_{\text {opt }}$ that maximizes $\rho_{1}(\mathbf{X})$. The controller implemented with this optimal realization $\mathbf{X}_{\text {opt }}$ needs a minimum bit length and has a maximum tolerance to the FWL error. This optimal controller realization problem is formally defined as

$$
v \triangleq \max _{\mathbf{X} \in \mathcal{S}_{C}} \rho_{1}(\mathbf{X}) .
$$

Assume that a controller has been designed using some standard controller design method. This controller, denoted as

$$
\mathbf{X}_{0} \triangleq\left[\begin{array}{cc}
\mathbf{D}_{C}^{0} & \mathbf{C}_{C}^{0} \\
\mathbf{B}_{C}^{0} & \mathbf{A}_{C}^{0}
\end{array}\right]
$$

is used as the initial controller realization in the above optimal controller realization problem. Let $\mathbf{p}_{0 i}$ be a right eigenvector of $\overline{\mathbf{A}}\left(\mathbf{X}_{0}\right)$ corresponding to the eigenvalue $\lambda_{i}$ and $\mathbf{y}_{0 i}$ be the reciprocal left eigenvector related to $\mathbf{p}_{0 i}$. The definition of $\mathcal{S}_{C}$ in (12) means that

$$
\mathbf{X} \triangleq \mathbf{X}(\mathbf{T})=\left[\begin{array}{cc}
\mathbf{I}_{l} & \mathbf{0} \\
\mathbf{0} & \mathbf{T}^{-1}
\end{array}\right] \mathbf{X}_{0}\left[\begin{array}{cc}
\mathbf{I}_{q} & \mathbf{0} \\
\mathbf{0} & \mathbf{T}
\end{array}\right]
$$

where $\operatorname{det} \mathbf{T} \neq 0$. It can then be shown that

$$
\overline{\mathbf{A}}(\mathbf{X})=\left[\begin{array}{cc}
\mathbf{I}_{m} & \mathbf{0} \\
\mathbf{0} & \mathbf{T}^{-1}
\end{array}\right] \overline{\mathbf{A}}\left(\mathbf{X}_{0}\right)\left[\begin{array}{cc}
\mathbf{I}_{m} & \mathbf{0} \\
\mathbf{0} & \mathbf{T}
\end{array}\right]
$$

which implies that

$$
\mathbf{p}_{i}=\left[\begin{array}{cc}
\mathbf{I}_{m} & \mathbf{0} \\
\mathbf{0} & \mathbf{T}^{-1}
\end{array}\right] \mathbf{p}_{0 i}, \quad \mathbf{y}_{i}=\left[\begin{array}{cc}
\mathbf{I}_{m} & \mathbf{0} \\
\mathbf{0} & \mathbf{T}^{\mathrm{T}}
\end{array}\right] \mathbf{y}_{0 i} .
$$

Hence

$$
\begin{aligned}
\mathbf{M}_{1}^{\mathrm{T}} \operatorname{Re}\left[\lambda_{i}^{*} \mathbf{y}_{i}^{*} \mathbf{p}_{i}^{\mathrm{T}}\right] \mathbf{M}_{2}^{\mathrm{T}} \\
=\left[\begin{array}{cc}
\mathbf{I}_{l} & \mathbf{0} \\
\mathbf{0} & \mathbf{T}^{\mathrm{T}}
\end{array}\right] \mathbf{M}_{1}^{\mathrm{T}} \operatorname{Re}\left[\lambda_{i}^{*} \mathbf{y}_{0 i}^{*} \mathbf{p}_{0 i}^{\mathrm{T}}\right] \mathbf{M}_{2}^{\mathrm{T}}\left[\begin{array}{cc}
\mathbf{I}_{q} & \mathbf{0} \\
\mathbf{0} & \mathbf{T}^{-\mathrm{T}}
\end{array}\right] \\
\triangleq\left[\begin{array}{cc}
\mathbf{I}_{l} & \mathbf{0} \\
\mathbf{0} & \mathbf{T}^{\mathrm{T}}
\end{array}\right] \mathbf{\Phi}_{i}\left[\begin{array}{cc}
\mathbf{I}_{q} & \mathbf{0} \\
\mathbf{0} & \mathbf{T}^{-\mathrm{T}}
\end{array}\right]
\end{aligned}
$$


with $\boldsymbol{\Phi}_{i}=\mathbf{M}_{1}^{\mathrm{T}} \operatorname{Re}\left[\lambda_{i}^{*} \mathbf{y}_{0 i}^{*} \mathbf{p}_{0 i}^{\mathrm{T}}\right] \mathbf{M}_{2}^{\mathrm{T}}$. Define the cost function

$$
\begin{aligned}
f(\mathbf{T}) \triangleq \max _{i \in\{1, \ldots, m+n\}} & \left(\frac{\left\|\left(\left[\begin{array}{cc}
\mathbf{I}_{l} & \mathbf{0} \\
\mathbf{0} & \mathbf{T}^{\mathrm{T}}
\end{array}\right] \mathbf{\Phi}_{i}\left[\begin{array}{cc}
\mathbf{I}_{q} & \mathbf{0} \\
\mathbf{0} & \mathbf{T}^{-\mathrm{T}}
\end{array}\right]\right) \circ \mathbf{X}(\mathbf{T})\right\|_{\text {sum }}}{\left|\lambda_{i}\right|\left(1-\left|\lambda_{i}\right|\right)}\right. \\
& \left.\times \log _{2} \frac{4\|\mathbf{X}(\mathbf{T})\|_{\max }}{g(\mathbf{X}(\mathbf{T}))}\right)
\end{aligned}
$$

In the above definition of the cost function $f(\mathbf{T})$

$$
\log _{2} \frac{4\|\mathbf{X}(\mathbf{T})\|_{\max }}{g(\mathbf{X}(\mathbf{T}))}
$$

is simply $\gamma(\mathbf{X})$ which estimates the cost of exponent bits, while

$$
\max _{i \in\{1, \ldots, m+n\}} \frac{\left\|\left(\left[\begin{array}{cc}
\mathbf{I}_{l} & \mathbf{0} \\
\mathbf{0} & \mathbf{T}^{\mathrm{T}}
\end{array}\right] \mathbf{\Phi}_{i}\left[\begin{array}{cc}
\mathbf{I}_{q} & \mathbf{0} \\
\mathbf{0} & \mathbf{T}^{-\mathrm{T}}
\end{array}\right]\right) \circ \mathbf{X}(\mathbf{T})\right\|_{\text {sum }}}{\left|\lambda_{i}\right|\left(1-\left|\lambda_{i}\right|\right)}
$$

is the inverse of $\mu_{1}(\mathbf{X})$ which estimates the cost of mantissa bits. Hence $f(\mathbf{T})$ can be used to measure the cost of total bits.

With the introduction of this cost function, the optimal controller realization problem (52) can then be posed as the optimization problem

$$
v^{-1}=\min _{\substack{\mathbf{T} \in \mathcal{R}^{n \times n} \\ \operatorname{det} \mathbf{T} \neq 0}} f(\mathbf{T}) .
$$

As the optimization problem (59) is highly non-linear, global optimization algorithms, such as the genetic algorithm (Man et al. 1998) and adaptive simulated annealing (Chen and Luk 1999), can be adopted to provide a (sub)optimal similarity transformation $\mathbf{T}_{\mathrm{opt}}$. Global optimization methods are, however, computationally demanding. Local optimization algorithms, such as Rosenbrock and Simplex algorithms (Beveridge and Schechter 1970), are computationally simpler but run more risks of only attaining a local solution. Our experience with the optimization problem (59) suggests that, unlike optimizing the mantissa measure $\mu_{1}(\mathbf{X})$ alone, the exponent measure $\gamma(\mathbf{X})$ in the criterion $\rho_{1}(\mathbf{X})$ helps to bound the solution set and the cost function $f(\mathbf{T})$ appears to behave better. It is also helpful to use some good initial controller realization, such as the openloop balanced realization (Laub et al. 1987), as the initial guess for the optimization routine. With $\mathbf{T}_{\text {opt }}$, the optimal realization $\mathbf{X}_{\text {opt }}$ can readily be computed.

\section{Numerical examples}

Two examples are used to illustrate the proposed design procedure for obtaining optimal FWL floatingpoint controller realizations and to compare it with the method given in Whidborne and Gu (2002). In the simulation, the bit length for implementing the state variables was sufficiently long that the second type of FWL error can be neglected.

Example 1: This example, taken from Gevers and $\mathrm{Li}$ (1993), has been studied by Whidborne and Gu (2002). The discrete-time plant is given by

$$
\begin{aligned}
\mathbf{A}_{P} & =\left[\begin{array}{cccc}
3.7156 e+0 & -5.4143 e+0 & 3.6525 e+0 & -9.6420 e-1 \\
1 & 0 & 0 & 0 \\
0 & 1 & 0 & 0 \\
0 & 0 & 1 & 0
\end{array}\right] \\
\mathbf{B}_{P} & =\left[\begin{array}{llll}
1 & 0 & 0 & 0
\end{array}\right]^{\mathrm{T}} \\
\mathbf{C}_{P} & =\left[\begin{array}{llll}
1.1160 e-6 & 4.3000 e-8 & 1.0880 e-6 & 1.4000 e-8
\end{array}\right] .
\end{aligned}
$$

The initial realization of the digital controller is given by

$$
\begin{aligned}
\mathbf{A}_{C}^{0} & =\left[\begin{array}{rrrr}
2.6743 e+0 & -5.7446 e+0 & 2.5101 e+0 & -9.1782 e-1 \\
2.8769 e-1 & -2.7446 e-2 & -6.9444 e-1 & -8.9358 e-3 \\
-3.3773 e-1 & 9.8699 e-1 & -3.2925 e-1 & -4.2367 e-3 \\
-8.3021 e-2 & -3.1988 e-3 & 9.1906 e-1 & -1.0415 e-3
\end{array}\right] \\
\mathbf{B}_{C}^{0} & =\left[\begin{array}{llll}
1.0959 e+6 & 6.3827 e+5 & 3.0262 e+5 & 7.4392 e+4
\end{array}\right]^{\mathrm{T}} \\
\mathbf{C}_{C}^{0} & =\left[\begin{array}{llll}
1.8180 e-1 & -2.8313 e-1 & 5.0006 e-2 & 6.1722 e-2
\end{array}\right], \\
\mathbf{D}_{C}^{0} & =0 .
\end{aligned}
$$

Based on the proposed FWL closed-loop stability measure, the optimization problem (59) was formed and solved using the MATLAB routine fminsearch.m, which is a local optimization search algorithm, to obtain an optimal transformation matrix

$\mathbf{T}_{\mathrm{opt}}=\left[\begin{array}{rrrr}7.7275 e+3 & -1.0904 e+2 & -2.1292 e+2 & 9.8042 e+1 \\ 6.9729 e+3 & 2.1370 e+3 & 4.4988 e+1 & 2.1812 e+2 \\ 6.2844 e+3 & 3.9092 e+3 & 2.9303 e+2 & 2.9240 e+2 \\ 5.5879 e+3 & 5.2862 e+3 & 5.5027 e+2 & 3.4367 e+2\end{array}\right]$

and the corresponding optimal realization of the digital controller $\mathbf{X}_{\text {opt }}$ given by

$$
\begin{aligned}
& \mathbf{A}_{C}^{\mathrm{opt}}=\left[\begin{array}{rrrr}
-1.4441 e+0 & -1.0500 e+0 & -6.0800 e-2 & -1.0102 e-1 \\
3.8412 e+0 & 2.4034 e+0 & 6.7143 e-2 & 1.7402 e-1 \\
-1.3159 e+1 & -4.5856 e+0 & 5.3403 e-1 & -6.8843 e-1 \\
3.2330 e-1 & -2.1078 e+0 & -6.6254 e-2 & 8.2322 e-1
\end{array}\right] \\
& \mathbf{B}_{C}^{\mathrm{opt}}=\left[\begin{array}{llll}
1.6342 e+2 & -2.5378 e+2 & 9.1370 e+2 & -6.1106 e-2
\end{array}\right]^{\mathrm{T}} \\
& \mathbf{C}_{C}^{\mathrm{opt}}=\left[\begin{array}{llll}
8.9770 e+1 & -1.0310 e+2 & -2.8290 e+0 & -8.0995 e+0
\end{array}\right], \\
& \mathbf{D}_{C}^{\mathrm{opt}}=0 .
\end{aligned}
$$

An 'optimal' controller realization problem was given in Whidborne and $\mathrm{Gu}$ (2002) based on the weighted closed-loop eigenvalue sensitivity index (48). We will use the index 's', rather then 'opt', to denote 
the solution of this 'optimal' controller realization problem. For this example, the transformation matrix solution obtained using the MATLAB routine fminsearch.m given in Whidborne and $\mathrm{Gu}(2002)$ is

$$
\mathbf{T}_{\mathrm{s}}=\left[\begin{array}{cccc}
8.1477 e+3 & 0 & 0 & 0 \\
7.0104 e+3 & 2.2671 e+3 & 0 & 0 \\
6.1991 e+3 & 3.9989 e+3 & 1.1558 e+2 & 0 \\
5.6761 e+3 & 5.2680 e+3 & 3.5814 e+2 & 1.5299 e+1
\end{array}\right]
$$

with the corresponding controller realization $\mathbf{X}_{\mathrm{s}}$ given by

$$
\begin{aligned}
\mathbf{A}_{C}^{\mathrm{s}} & =\left[\begin{array}{rrrr}
-9.9795 e-1 & -9.5988 e-1 & -4.7357 e-3 & -1.7234 e-3 \\
2.1137 e+0 & 1.6951 e+0 & -2.2171 e-2 & 5.2689 e-3 \\
-1.4177 e+0 & 6.1144 e-1 & 6.7870 e-1 & -9.0420 e-2 \\
1.9428 e+0 & -2.4577 e+0 & 4.2234 e-1 & 9.4079 e-1
\end{array}\right] \\
\mathbf{B}_{C}^{\mathrm{s}} & =\left[\begin{array}{llll}
1.3451 e+2 & -1.3439 e+2 & 5.3833 e+1 & -2.5633 e+1
\end{array}\right]^{\mathrm{T}} \\
\mathbf{C}_{C}^{\mathrm{s}} & =\left[\begin{array}{llll}
1.5673 e+2 & -1.1677 e+2 & 2.7885 e+1 & 9.4430 e-1
\end{array}\right], \\
\mathbf{D}_{C}^{\mathrm{s}} & =0 .
\end{aligned}
$$

It is obvious that the true minimum exponent bit length $\beta_{e}^{\min }$ for a realization $\mathbf{X}$ can directly be obtained by examining the elements of $\mathbf{X}$. The true minimum mantissa bit length $\beta_{w}^{\min }$, however, can only be obtained through simulation. That is, starting from a very large $\beta_{w}$, reduce $\beta_{w}$ by one bit and check the closed-loop stability. The process is repeated until there appears closed-loop instability at $\beta_{w}=\beta_{w u}$. Then $\beta_{w}^{\min }=$ $\beta_{w u}+1$. Table 1 summarizes the various measures, the corresponding estimated minimum bit lengths and the true minimum bit lengths for the three controller realizations $\mathbf{X}_{0}, \mathbf{X}_{\mathrm{s}}$ and $\mathbf{X}_{\mathrm{opt}}$, respectively. It can be seen that the floating-point implementation of $\mathbf{X}_{0}$ needs at least 26 bits (20 mantissa bits and five exponent bits) while the implementation of $\mathbf{X}_{\text {opt }}$ needs at least 13 bits (eight mantissa bits and four exponent bits). The reduction in the bit length required is 13 (12-bit reduction for the mantissa part and 1-bit reduction for the exponent part). Comparing $\mathbf{X}_{\mathrm{opt}}$ with $\mathbf{X}_{\mathrm{s}}$, it can be seen that $\mathbf{X}_{\text {opt }}$ needs one bit less in the exponent part and one bit less in the mantissa part.

Note that any realization $\mathbf{X} \in \mathcal{S}_{C}$ implemented in infinite precision will achieve the exact performance of the infinite-precision implemented $\mathbf{X}_{0}$, which is the designed controller performance. For this reason, the infinite-precision implemented $\mathbf{X}_{0}$ is referred to as the ideal controller realization $\mathbf{X}_{\text {ideal }}$. Figure 2 compares the unit impulse response of the plant output $y(k)$ for the ideal controller $\mathbf{X}_{\text {ideal }}$ with those of the 8-mantissa-bit plus 5-exponent-bit implemented $\mathbf{X}_{\mathrm{s}}$ and 8-mantissa-bit plus 4-exponent-bit implemented $\mathbf{X}_{\text {opt }}$. The 8-mantissabit implemented $\mathbf{X}_{0}$ quickly becomes unstable and is not shown here. From figure 2, it can be seen that the closedloop system with the 13-bit implemented $\mathbf{X}_{\text {opt }}$ is stable while the system with the 14-bit implemented $\mathbf{X}_{\mathrm{s}}$ is unstable. Figure 3 compares the unit impulse response of $y(k)$ for $\mathbf{X}_{\text {ideal }}$ with those of the 9-mantissa-bit plus 5-exponent-bit implemented $\mathbf{X}_{\mathrm{s}}$ and the 9-mantissabit plus 4-exponent-bit implemented $\mathbf{X}_{\text {opt }}$. Again the 9-mantissa-bit implemented $\mathbf{X}_{0}$ is unstable and is not shown. It can be seen that the response with the 14-bit implemented $\mathbf{X}_{\mathrm{opt}}$ is clearly closer to the ideal performance than that of the 15-bit implemented $\mathbf{X}_{\mathrm{s}}$.

Example 2: This example is taken from a continuoustime $H_{\infty}$ robust control example studied in Keel and Bhattacharryya (1997) and Whidborne et al. (2001). The continuous-time plant model and $H_{\infty}$ controller are sampled with a sampling period of $4 \mathrm{~ms}$ to obtain the discrete-time plant

$$
\begin{aligned}
\mathbf{A}_{P} & =\left[\begin{array}{cc}
1.9980 e+0 & -9.9800 e-1 \\
1 & 0
\end{array}\right] \\
\mathbf{B}_{P} & =\left[\begin{array}{ll}
1 & 0
\end{array}\right]^{\mathrm{T}}, \quad \mathbf{C}_{P}=\left[\begin{array}{ll}
3.9880 e-3 & -4.0040 e-3
\end{array}\right]
\end{aligned}
$$

and the initial realization of the digital controller

$$
\begin{aligned}
\mathbf{A}_{C}^{0} & =\left[\begin{array}{ccc}
2.3985 e+0 & -1.8017 e+0 & 4.0317 e-1 \\
1 & 0 & 0 \\
0 & 1 & 0
\end{array}\right] \\
\mathbf{B}_{C}^{0} & =\left[\begin{array}{lll}
1 & 0 & 0
\end{array}\right]^{\mathrm{T}} \\
\mathbf{C}_{C}^{0} & =\left[\begin{array}{lll}
-7.3591 e+1 & 1.4661 e+2 & -7.3018 e+1
\end{array}\right], \\
\mathbf{D}_{C}^{0} & =1.2450 e+2 .
\end{aligned}
$$

The MATLAB routine fminsearch.m was used to solve the optimization problem based on the FWL

\begin{tabular}{lccccccccc}
\hline Realization & $\rho_{1}$ & $\hat{\beta}_{1}^{\min }$ & $\mu_{1}$ & $\hat{\beta}_{w 1}^{\min }$ & $\gamma$ & $\hat{\beta}_{e}^{\min }$ & $\beta^{\min }$ & $\beta_{w}^{\min }$ & $\beta_{e}^{\min }$ \\
\hline $\mathbf{X}_{0}$ & $2.6644 \mathrm{e}-9$ & 30 & $8.5182 \mathrm{e}-8$ & 23 & $3.1971 \mathrm{e}+1$ & 5 & 26 & 20 & 5 \\
$\mathbf{X}_{\mathrm{s}}$ & $4.7588 \mathrm{e}-6$ & 19 & $8.7907 \mathrm{e}-5$ & 13 & $1.8473 \mathrm{e}+1$ & 5 & 15 & 9 & 5 \\
$\mathbf{X}_{\text {opt }}$ & $9.5931 \mathrm{e}-6$ & 18 & $1.5229 \mathrm{e}-4$ & 12 & $1.5875 \mathrm{e}+1$ & 4 & 13 & 8 & 4 \\
\hline
\end{tabular}

Table 1. Various measures, corresponding estimated minimum bit lengths and true minimum bit lengths for three controller realizations $\mathbf{X}_{0}, \mathbf{X}_{\mathrm{s}}$ and $\mathbf{X}_{\text {opt }}$ of Example 1. 


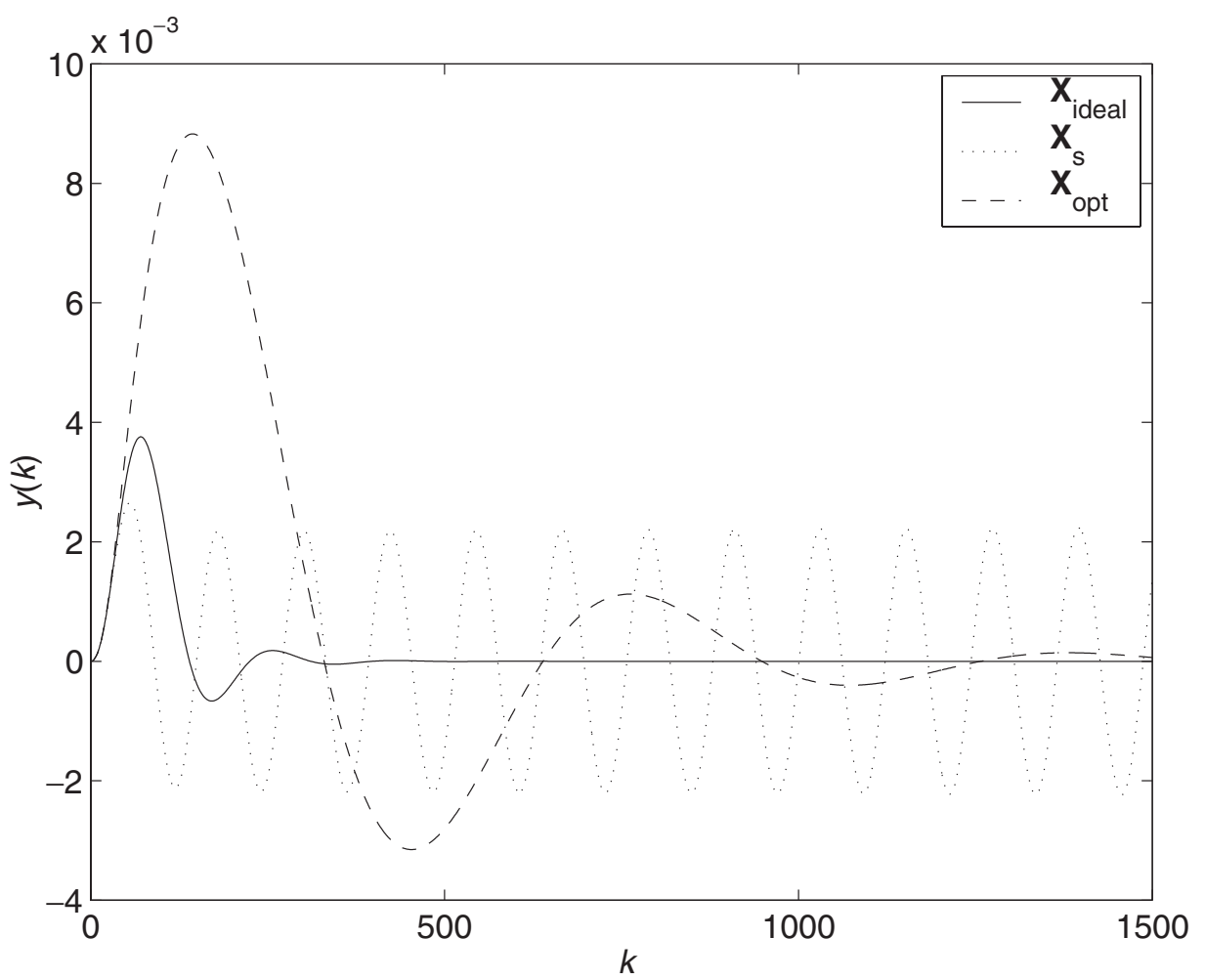

Figure 2. Unit impulse response $y(k)$ for $\mathbf{X}_{\text {ideal }}$, 14-bit implemented $\mathbf{X}_{\mathrm{s}}$ (eight mantissa bits and five exponent bits) and 13-bit implemented $\mathbf{X}_{\text {opt }}$ (eight mantissa bits and four exponent bits) of Example 1.

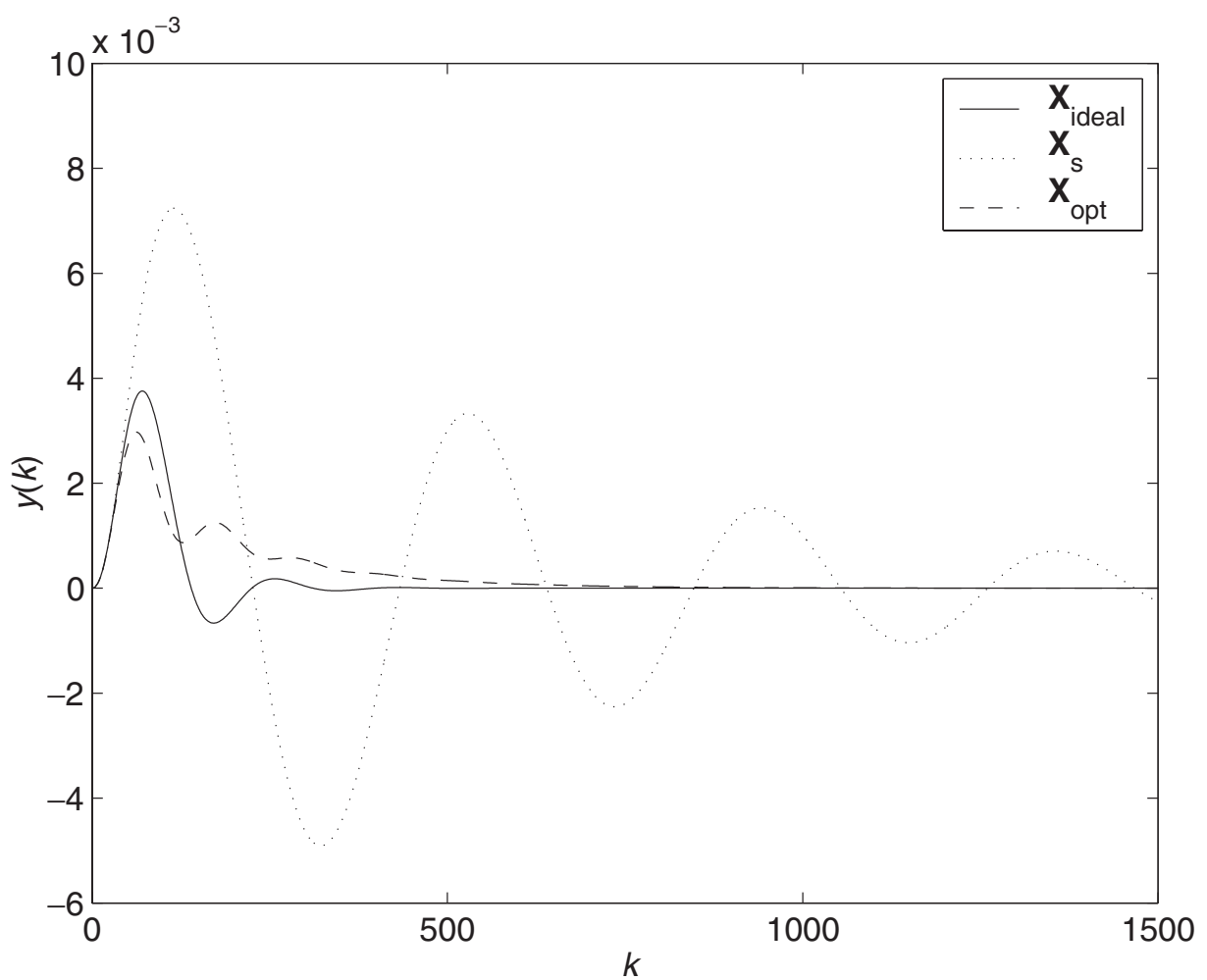

Figure 3. Unit impulse response $y(k)$ for $\mathbf{X}_{\text {ideal }}$, 15-bit implemented $\mathbf{X}_{\mathrm{s}}$ (nine mantissa bits and five exponent bits) and 14-bit implemented $\mathbf{X}_{\mathrm{opt}}$ (nine mantissa bits and four exponent bits) of Example 1. 
closed-loop stability measure presented in this paper to obtain an optimal transformation matrix

$$
\mathbf{T}_{\mathrm{opt}}=\left[\begin{array}{rrr}
1.8515 e+2 & 7.2829 e-1 & 9.7266 e+0 \\
1.8540 e+2 & 1.6951 e+1 & -2.3477 e+0 \\
1.8566 e+2 & 3.3300 e+1 & -1.4508 e+1
\end{array}\right]
$$

and the corresponding optimal realization of the digital controller $\mathbf{X}_{\text {opt }}$ with

$$
\begin{aligned}
& \mathbf{A}_{C}^{\mathrm{opt}}=\left[\begin{array}{rrc}
1.0006 e+0 & -8.8718 e-2 & 9.9092 e-2 \\
-2.7168 e-2 & 1.0178 e+0 & -4.5738 e-1 \\
-3.6546 e-2 & 3.2513 e-2 & 3.8007 e-1
\end{array}\right] \\
& \mathbf{B}_{C}^{\mathrm{opt}}=\left[\begin{array}{lll}
-6.8999 e+0 & 9.2711 e+1 & 1.2450 e+2
\end{array}\right]^{\mathrm{T}} \\
& \mathbf{C}_{C}^{\mathrm{opt}}=\left[\begin{array}{lll}
-3.6469 e-2 & 2.7168 e-2 & -6.1334 e-1
\end{array}\right], \\
& \mathbf{D}_{C}^{\mathrm{opt}}=1.2450 e+2 .
\end{aligned}
$$

Based on the method of the weighted closed-loop eigenvalue sensitivity index (Whidborne and $\mathrm{Gu}$ 2002), the MATLAB routine fminsearch.m found a transformation matrix solution

$$
\mathbf{T}_{\mathrm{s}}=\left[\begin{array}{ccc}
1.8446 e+2 & 0 & 0 \\
1.8500 e+2 & 2.9433 e+0 & 0 \\
1.8553 e+2 & 5.9061 e+0 & 8.3753 e-3
\end{array}\right]
$$

with the corresponding controller realization $\mathbf{X}_{\mathrm{s}}$ given by

$$
\begin{aligned}
\mathbf{A}_{C}^{\mathrm{s}} & =\left[\begin{array}{rrr}
9.9711 e-1 & -1.5840 e-2 & 1.8305 e-5 \\
3.2077 e-5 & 9.9558 e-1 & -1.1505 e-3 \\
-2.8762 e-2 & 2.5216 e-1 & 4.0584 e-1
\end{array}\right] \\
\mathbf{B}_{C}^{\mathrm{s}} & =\left[\begin{array}{lll}
5.4211 e-3 & -3.4074 e-1 & 1.2019 e+2
\end{array}\right]^{\mathrm{T}} \\
\mathbf{C}_{C}^{\mathrm{s}} & =\left[\begin{array}{lll}
-2.9785 e-2 & 2.6087 e-1 & -6.1154 e-1
\end{array}\right], \\
\mathbf{D}_{C}^{\mathrm{s}} & =1.2450 e+02 .
\end{aligned}
$$

Table 2 summarizes the various measures, the corresponding estimated minimum bit lengths and the true minimum bit lengths for $\mathbf{X}_{0}, \mathbf{X}_{\mathrm{s}}$ and $\mathbf{X}_{\mathrm{opt}}$. Obviously, the implementation of $\mathbf{X}_{0}$ needs at least 30 bits (25 mantissa bits and four exponent bits) while the implementation of $\mathbf{X}_{\mathrm{opt}}$ requires at least 12 bits (seven mantissa bits and four exponent bits). It can be seen that the optimization results in a reduction of 18 bits for the mantissa part. It is interesting to note that the realization $\mathbf{X}_{\mathrm{s}}$, while reducing 16 bits in the required $\beta_{w}^{\min }$, actually increases the required $\beta_{e}^{\min }$ by one bit, compared with $\mathbf{X}_{0}$. This is not surprising, since the measure $r(\mathbf{X})$ completely neglects the exponent part. Figure 4 compares the unit impulse response of the plant output $y(k)$ for the ideal controller $\mathbf{X}_{\text {ideal }}$ with those of the 14-bit implemented $\mathbf{X}_{\mathrm{s}}$ (eight mantissa bits and five exponent bits) and the 14-bit implemented $\mathbf{X}_{\text {opt }}$ (nine mantissa bits and four exponent bits). It can be seen that the closed-loop system with the 14-bit implemented $\mathbf{X}_{\text {opt }}$ is stable while the system with the 14-bit implemented $\mathbf{X}_{\mathrm{s}}$ is unstable. Figure 5 compares the unit impulse response of $y(k)$ for $\mathbf{X}_{\text {ideal }}$ with those of the 15bit implemented $\mathbf{X}_{\mathrm{s}}$ (nine mantissa bits and five exponent bits) and the 15-bit implemented $\mathbf{X}_{\text {opt }}$ (ten mantissa bits and four exponent bits). The performance of the 15bit implemented $\mathbf{X}_{\text {opt }}$ is clearly closer to the ideal performance than that of the 15-bit implemented $\mathbf{X}_{\mathrm{s}}$.

\section{Brief discussion on the direct approach}

A limitation of the indirect strategy, one may argue, is that it relies on a fixed control law or transfer function. The direct approach removes this assumption and appears to be a better approach in dealing with the FWL issues. Apart from the excellent work by Liu et al. (1992), we are only aware of another case of successfully adopting a direct strategy (Yang et al. 2000), where the standard $H_{\infty}$ control design was extended to include FWL controller parameter perturbations, and a Riccati inequality approach was developed to directly obtain optimal controller realizations satisfying both the $H_{\infty}$ robustness and FWL closed-loop stability requirements. Except for $H_{\infty}$ and LQG, it seems to be very difficult to extend various controller design methods to this direct strategy. The indirect approach, however, is very flexible. Controller synthesis is generally a highly complicated task, involving many trade-offs for various conflicting requirements. Even when a direct method can be found, the indirect approach is still useful, as it can be used to further optimize a controller realization obtained with the direct approach.

To see where the difficulties are for the direct approach, let us discuss how to extend the work of

\begin{tabular}{llclrcrrrr}
\hline Realization & \multicolumn{1}{c}{$\rho_{1}$} & $\hat{\beta}_{1}^{\min }$ & \multicolumn{1}{c}{$\mu_{1}$} & $\hat{\beta}_{w 1}^{\min }$ & $\gamma$ & $\hat{\beta}_{e}^{\min }$ & $\beta^{\min }$ & $\beta_{w}^{\min }$ & $\beta_{e}^{\min }$ \\
\hline $\mathbf{X}_{0}$ & $2.6767 \mathrm{e}-11$ & 37 & $2.8122 \mathrm{e}-10$ & 31 & $1.0506 \mathrm{e}+1$ & 4 & 30 & 25 & 4 \\
$\mathbf{X}_{\mathrm{s}}$ & $3.1047 \mathrm{e}-6$ & 20 & $7.6679 \mathrm{e}-5$ & 13 & $2.4697 \mathrm{e}+1$ & 5 & 15 & 9 & 5 \\
$\mathbf{X}_{\text {opt }}$ & $5.8446 \mathrm{e}-6$ & 19 & $8.2771 \mathrm{e}-5$ & 13 & $1.4162 \mathrm{e}+1$ & 4 & 12 & 7 & 4 \\
\hline
\end{tabular}

Table 2. Various measures, corresponding estimated minimum bit lengths and true minimum bit lengths for three controller realizations $\mathbf{X}_{0}, \mathbf{X}_{\mathrm{s}}$ and $\mathbf{X}_{\text {opt }}$ of Example 2. 


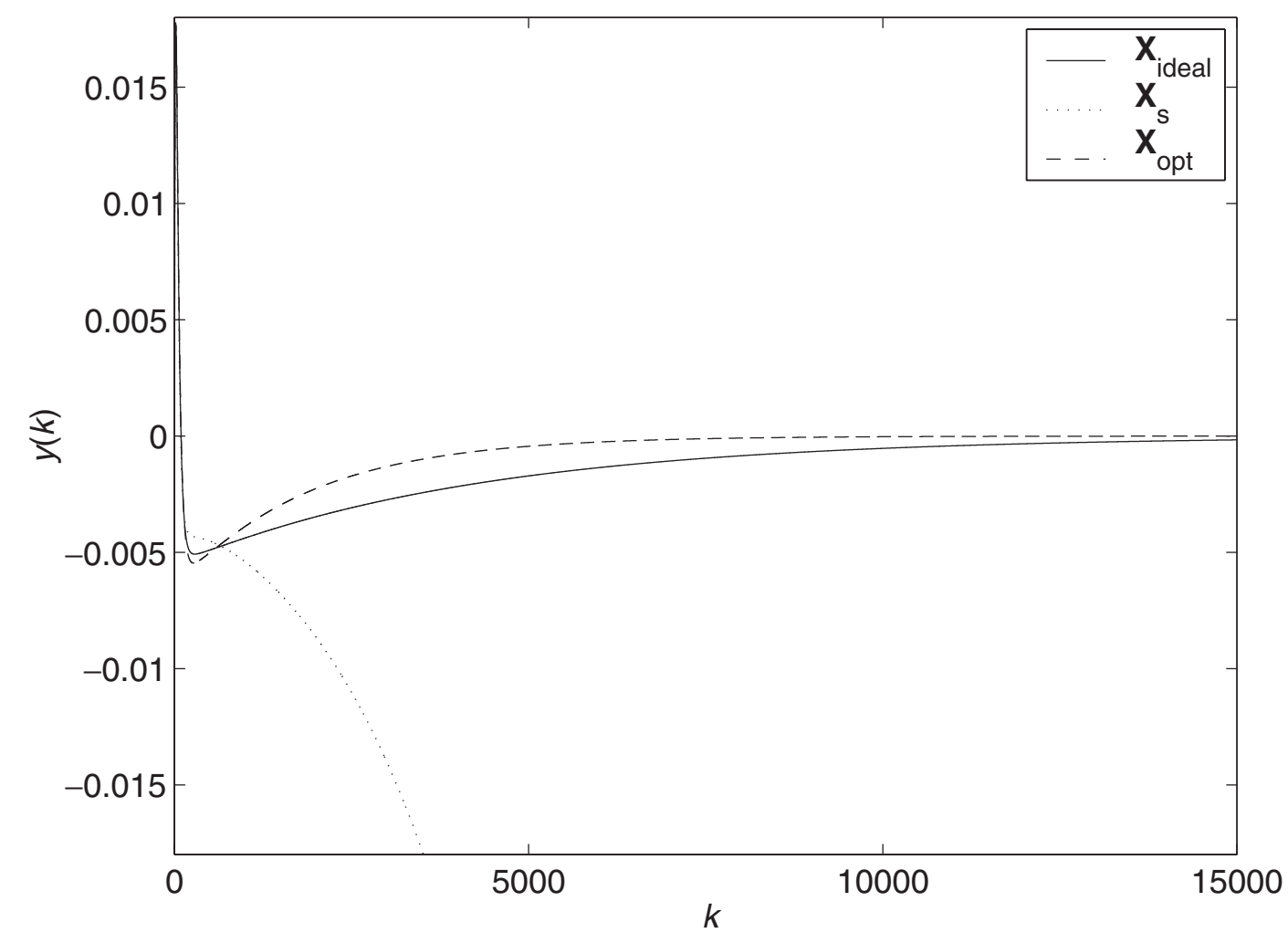

Figure 4. Unit impulse response $y(k)$ for $\mathbf{X}_{\text {ideal }}$, 14-bit implemented $\mathbf{X}_{\mathrm{s}}$ (eight mantissa bits and five exponent bits) and 14-bit implemented $\mathbf{X}_{\mathrm{opt}}$ (nine mantissa bits and four exponent bits) of Example 2.

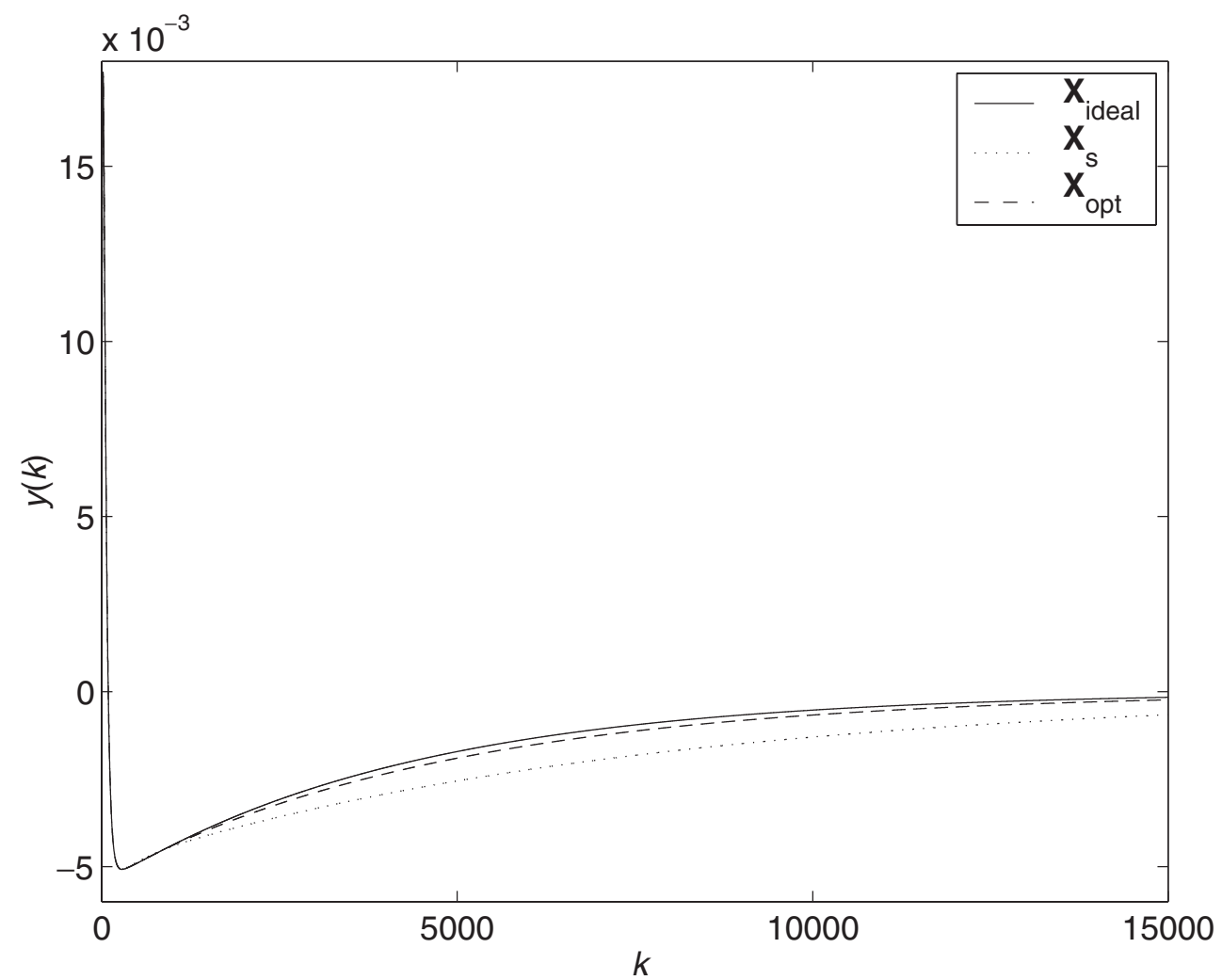

Figure 5. Unit impulse response $y(k)$ for $\mathbf{X}_{\text {ideal }}$, 15-bit implemented $\mathbf{X}_{\mathrm{s}}$ (nine mantissa bits and five exponent bits) and 15-bit implemented $\mathbf{X}_{\mathrm{opt}}$ (ten mantissa bits and four exponent bits) of Example 2. 
Liu et al. (1992) to the generic setting. First define the controller realization set

$$
\begin{aligned}
\mathcal{U}_{C} \triangleq & \left\{\mathbf{X} \mid \mathbf{X} \in \mathcal{R}^{(l+n) \times(q+n)},\right. \\
& \mathbf{X} \text { is a controller realization stabilizing } P(z)\} .
\end{aligned}
$$

Assume that a performance index can be formulated to reflect the needs of all the performance requirements, including FWL implementation considerations. Extending the idea of Liu et al. (1992) to this generic setting, the optimization problem $\uparrow$ for FWL controller realization design can be defined as

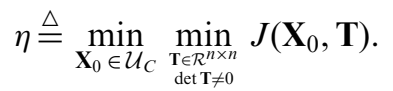

The cost function

$$
J\left(\mathbf{X}_{0}, \mathbf{T}\right) \triangleq \lim _{k \rightarrow \infty} \mathcal{E}\left[\mathbf{y}^{\mathrm{T}}(k) \mathbf{Q} \mathbf{y}(k)+\mathbf{u}^{\mathrm{T}}(k) \mathbf{R} \mathbf{u}(k)\right]
$$

depends on $\mathbf{X}_{0}$ and $\mathbf{T}$, where $\mathcal{E}[\cdot]$ represents the average value, $\mathbf{y}(k)$ is the output of $P(z), \mathbf{u}(k)$ is the output of $C(z), \mathbf{Q}$ and $\mathbf{R}$ are given matrices. It is easy to see that the problem (61) can be broken into two parts and solved with the two coupling optimization problems:

$$
\begin{gathered}
\xi\left(\mathbf{X}_{0}\right) \triangleq \min _{\substack{\mathbf{T} \in \mathcal{R}^{n \times n} \\
\operatorname{det} \mathbf{T} \neq 0}} J\left(\mathbf{X}_{0}, \mathbf{T}\right) \\
\eta=\min _{\mathbf{X}_{0} \in \mathcal{U}_{C}} \xi\left(\mathbf{X}_{0}\right) .
\end{gathered}
$$

Providing that the optimization problem (63) can be solved exactly, for example, some close-form solution of the problem (63) can be obtained, the optimization problem (64) can be tackled and hopefully solved successfully. Apart from a few performance cost functions, how to solve the generic optimization problem (61) is still an open problem. It is also clear that the first part (63) of the optimization problem (61) has the same form as our optimization problem (59). Thus, the studies on optimal realizations for a fixed control law, like the one in this paper, may provide useful insights to help solve the more generic optimal realization problem (61).

\section{Conclusions}

The closed-loop stability issue of finite-precision realizations has been investigated for digital controller implemented in floating-point arithmetic. A new computationally tractable FWL closed-loop stability measure has been derived for floating-point controller realizations. Unlike the existing methods, which only consider

$\dagger$ There appeared $\beta_{i}$ (the fractional wordlength storing state variable) in the original problem of Liu et al. (1992). We omit $\beta_{i}$ here as it has no relevance to our discussion. the mantissa part of the floating-point scheme, the proposed measure takes into account both the exponent and mantissa parts of the floating-point format. It has been shown that this new measure yields a more accurate estimate for the FWL closed-loop stability. Based on this FWL closed-loop stability measure, the optimal controller realization problem has been formulated, which can then be solved using numerical optimization algorithms. Two numerical examples have demonstrated that the proposed design procedure yields computationally efficient controller realizations suitable for FWL floating-point implementation in realtime applications. The idea of considering both the dynamic range and precision of FWL floating-point arithmetic is generic and can be used to deal with the similar problems in FWL fixed-point arithmetic and FWL block-floating-point arithmetic. In fact, the implementation of a digital controller should include not only the selection of realizations but also the choice of number representation formats. Further research is currently being conducted to develop the design procedure for choosing an optimal controller realization as well as an appropriate representation scheme for a given control law to achieve the best performance and computational efficiency.

\section{Acknowledgements}

J. Wu and S. Chen wish to acknowledge the support of the United Kingdom Royal Society under a KC Wong fellowship (RL/ART/CN/XFI/KCW/11949). J. $\mathrm{Wu}$ wishes to acknowledge the support of the National Natural Science Foundation of China under Grants 60174026 and 60374002.

\section{References}

BAuer, P. H., 1995, Absolute response error bounds for floating point digital filters in state space representation. IEEE Transactions on Circuits Systems II, 42, 610-613.

BAuer, P. H., and WANG, J., 1993, Limit cycle bounds for floating point implementations of second-order recursive digital filters. IEEE Transactions on Circuits Systems II, 40, 493-501.

Beveridge, G. S. G., and Schechter, R. S., 1970, Optimization: Theory and Practice (McGraw-Hill).

Chen, S., and Luk, B. L., 1999, Adaptive simulated annealing for optimization in signal processing applications. Signal Processing, 79, 117-128.

Chen, S., Wu, J., Istepanian, R. S. H., and Chu, J., 1999, Optimizing stability bounds of finite-precision PID controller structures. IEEE Transactions on Automatic Control, 44, 2149-2153.

Fialho, I. J., and Georgiou, T .T., 1994, On stability and performance of sampled-data systems subject to wordlength constraint. IEEE Transactions on Automatic Control, 39, 2476-2481. 
Fialho, I. J., and Georgiou, T. T., 2001, Computational algorithms for sparse optimal digital controller realizations. In R. S. H. Istepanian and J. F. Whidborne (Eds) Digital Controller Implementation and Fragility: $A$ Modern Perspective (London: Springer Verlag), pp. 105-121.

Gevers, M., and Li, G., 1993, Parameterizations in Control, Estimation and Filtering Problems: Accuracy Aspects (London: Springer Verlag).

Istepanian, R. S. H., and Whidborne, J. F. (Eds), 2001, Digital Controller Implementation and Fragility: A Modern Perspective (London: Springer Verlag).

Istepanian, R. S. H., Whidborne, J. F., and Bauer, P., 2000, Stability analysis of block floating point digital controllers. In Proceedings of the UKACC International Conference on Control 2000, Cambridge, UK, CD-ROM, 6 pages.

Kalliojärvi, K., and Astola, J., 1996, Roundoff errors in block-floating-point systems. IEEE Transactions on Signal Processing, 44, 783-790.

KAnEKo, T., 1973, Limit-cycle oscillations in floating digital filters. IEEE Transactions on Audio Electroacoustics, 21, $100-106$.

Keel, L. H., and Bhattacharryya, S. P., 1997, Robust, fragile, or optimal? IEEE Transactions on Automatic Control, 42, 1098-1105.

Laub, A. J., Heath, M. T., Paige, C. C., and Ward, R. C., 1987, Computation of system balancing transformations and other applications of simultaneous diagonalization reduction algorithms. IEEE Transactions on Automatic Control, 32, 115-122.

LI, G., 1998, On the structure of digital controllers with finite word length consideration. IEEE Transactions on Automatic Control, 43, 689-693.

Li, G., and Gevers, M., 1990, Optimal finite precision implementation of a state-estimate feedback controller. IEEE Transactions on Circuits and Systems, CAS-38, 1487-1499.

Li, G., Wu, J., Chen, S., and Zhao, K. Y., 2002, Optimum structures of digital controllers in sampled-data systems: a roundoff noise analysis. IEE Proceedings of the Control Theory and Applications, 149, 247-255.

LiU, B., and KANEKo, T., 1969, Error analysis of digital filters realized with floating point arithmetic. Proceedings of the IEEE, 57, 1735-1747.

LiU, K., Skelton, R., and Grigoriadis, K., 1992, Optimal controllers for finite wordlength implementation. IEEE Transactions on Automatic Control, 37, 1294-1304.

Madievski, A. G., Anderson, B. D. O., and Gevers, M., 1995, Optimum realizations of sampled-data controllers for FWL sensitivity minimization. Automatica, 31, 367-379.

Man, K. F., Tang, K. S., and Kwong, S., 1998, Genetic Algorithms: Concepts and Design (London: SpringerVerlag).

Miller, R. K., Michel, A. N., and Farrell, J. A., 1989, Quantizer effects on steady-state error specifications of digital feedback control systems. IEEE Transactions on Automatic Control, 34, 651-654.

Miller, R. K., Mousa, M. S., and Michel, A. N., 1988, Quantization and overflow effects in digital implementations of linear dynamic controllers. IEEE Transactions on Automatic Control, 33, 698-704.

Molchanov, A. P., and Bauer, P. H., 1995, Robust stability of digital feedback control systems with floating point arithmetic. In Proceedings of the 34th IEEE International Conference on Decision and Control, New Orleans, LA, USA, pp. 4251-4258.

Moroney, P., Willsky, A. S., and Houpt, P. K., 1980, The digital implementation of control compensators: the coefficient wordlength issue. IEEE Transactions on Automatic Control, AC-25, 621-630.

Ralev, K. R., and Bauer, P. H., 1999, Realization of block floating-point digital filters and application to block implementations. IEEE Transactions on Signal Processing, 47, 1076-1086.

RaO, B. D., 1996, Roundoff noise in floating point digital filters. Control and Dynamic Systems, 75, 79-103.

Rink, R. E., and Chong, H. Y., 1979, Performance of state regulator systems with floating point computation. IEEE Transactions on Automatic Control, 24, 411-421.

Whidborne, J. F., and Gu, D.-W., 2002, Optimal finiteprecision controller and filter implementations using floating-point arithmetic. In Proceedings of the 15th IFAC World Congress, Barcelona, Spain, CD-ROM, Paper 990.

Whidborne, J. F., Istepanian, R. S. H., and Wu, J., 2001, Reduction of controller fragility by pole sensitivity minimization. IEEE Transactions on Automatic Control, 46, $320-325$.

Whidborne, J. F., Wu, J., and Istepanian, R. S. H., 2000, Finite word length stability issues in an $l_{1}$ framework. International Journal of Control, 73, 166-176.

Williamson, D., and Kadiman, K., 1989, Optimal finite wordlength linear quadratic regulation. IEEE Transactions on Automatic Control, 34, 1218-1228.

Wu, J., Chen, S., LI, G., and Chu, J., 2001 b, Finite word length implementation for digital reduced order observer based controllers. Developments in Chemical Engineering and Mineral Processing, 9, 41-48.

Wu, J., Chen, S., Li, G., Istepanian, R. S. H., and Chu, J., 2000, Shift and delta operator realizations for digital controllers with finite-word-length considerations. IEE Proceedings of Control Theory and Applications, 147, 664-672.

Wu, J., Chen, S., Li, G., Istepanian, R. S. H., and Chu, J., 2001 a, An improved closed-loop stability related measure for finite-precision digital controller realizations. IEEE Transactions on Automatic Control, 46, 1162-1166.

YANG, G.-H., WANG, J. L., and LIN, C., 2000, $H_{\infty}$ control for linear systems with additive controller gain variations. International Journal of Control, 73, 1500-1506. 